\title{
疫情背景下多元化素材在分析化学线上教学中的综合运用
}

王敏*

浙江大学化学系, 杭州 310058

摘要: 受新冠肺炎(COVID-19)疫情的影响, 笔者在 2019-2020 春夏学期承担的 “分析化学 II' 课程以网络教学的方式 展开。在线上教学过程中, 借助网络上丰富的信息, 综合运用多种多媒体素材, 特别是与新冠肺炎的检测方法、治疗方 案、药物开发等密切相关的信息和知识点, 融入分析化学的教学过程和课程思政, 发挥线上教学的优势, 为线上线下一 流课程的建设提供思路和实践。

关键词: 线上教学; 分析化学; 课程思政; 新冠肺炎

中图分类号: G64; O6

\section{Comprehensive Utilization of Diversified Materials in Analytical Chemistry Teaching under the Background of COVID-19}

\author{
Min Wang * \\ Department of Chemistry, Zhejiang University, Hangzhou 310058, P. R. China.
}

Abstract: Because of the outbreak of COVID-19, the course 'Analytical Chemistry II' is taught via online for the 2019-2020 spring semester. During the process, materials from various sources are successfully incorporated into the teaching. Particularly, the detection, therapy, drug development for COVID-19 are all tightly related to analytical chemistry. With introduction of these correlative multimedia materials into the teaching and learning practice, we can help the students better understand the concept of analytical chemistry. It is a beneficial attempt for combination of online and offline teaching in the future.

Key Words: Online teaching; Analytical chemistry; Ideological teaching; COVID-19

\section{1 引言}

浙江大学化学专业培养方案中, 分析化学课程分为 I 和 II。其中 “分析化学 I” (32 学时, 2 学 分)在大二秋冬学期开设, 除了传统的化学分析的内容外, 还包含分光光度法和电位法等仪器分析方 法; “分析化学 II” (48 学时, 3 学分)在大二春夏学期开设, 以常见的仪器分析方法为主要内容。 “分析化学 II” 同时也是一门双语教学课程, 近年来每年选课人数 90-100。2019 年, 分析化学课程 被认定为浙江省线下一流课程。

疫情 “迫使” 我们进入全面网络授课。新冠肺炎的诊断和治疗, 与化学学科, 特别是分析化学 密切相关。在本学期 “分析化学 II” 线上授课的过程中, 我们综合利用各种多媒体素材丰富课程内 容, 将新冠肺炎相关的信息和知识点与课程充分结合。本文将以围绕新冠肺炎的导论课、奎宁为出 发点的课程思政以及抗疫过程中新技术的运用等为例介绍这门课的课程设计。 


\section{2 以新冠肺炎诊断为切入点, 上好导论课}

二月初开始准备网课之时, 正值 “战疫” 胶着阶段, 网上与疫情相关的文章不断出现, 尤其是 一些 “核酸检测阴转阳” “治愈者出院 10 天后复检核酸阳性” 等报道, 进一步增大了人们对 “新 冠确诊” 的疑虑。引导学生以科学的态度面对疫情中的各种问题, 是教师的重要职责之一。

由此, 在通常 1 个学时的导论课中, 我们增加了问题导向的微课 “从新冠疫情的爆发看分析化 学的重要性”, 将 COVID-19 诊断这一现实紧迫问题与分析化学理论和方法紧密结合。2 月 7 日, 新浪科技 “科学大家” 栏目出品文章 “知已知彼：关于病毒, 这些秘密你必须知道” ${ }^{[1]} ; 2$ 月 10 日, 大连理工大学虚拟实验平台(Moolsnet)发布文章 “浅谈 2019-nCoV 新冠状病毒的核酸检测方法” [2]; 2 月 18 日中国新闻社的微信推文 “方舱医院的生活什么样? 网友: 太高能了!” [3]中有一段视频 “新 冠肺炎患者的自述：我在方舱医院的一天” , 其中有一个环节是血氧饱和度的现场快速检测。结合 国家卫生健康委员会于 2 月 19 日发布的《新型冠状病毒肺炎诊疗方案(试行第六版)》 ${ }^{[4]}$ 中的诊断标 准，这几篇时效性推文成为这一微课的主要素材来源。

图 1 展示了这个微课的教学设计。从大家普遍关心和纠结的问题 “如果现在有点感冒发烧, 要 不要去医院” 出发, 引出血氧饱和度的概念; 以血氧饱和度的测量为例, 从最初的实验室仪器到现 在的连续无损伤血氧测量仪, 同学们可以直观地感受仪器分析便携化、小型化、及时性、现场快速 检测的发展趋势, 体会到分析化学的应用和发展不仅仅停留在实验室中, 而是与我们生活质量的提 升息息相关。围绕新冠肺炎的确诊挑战, 简单介绍了核酸检测的几种方法, 使同学们对其有初步的 了解, 并对分析化学在生命科学和临床医学领域的重要性有更深刻的认识。最终以分析化学的发展 方向作为这个微课的总结, 自然转入到我们这个课程的目的以及下一节内容 “光学分析方法导论”。

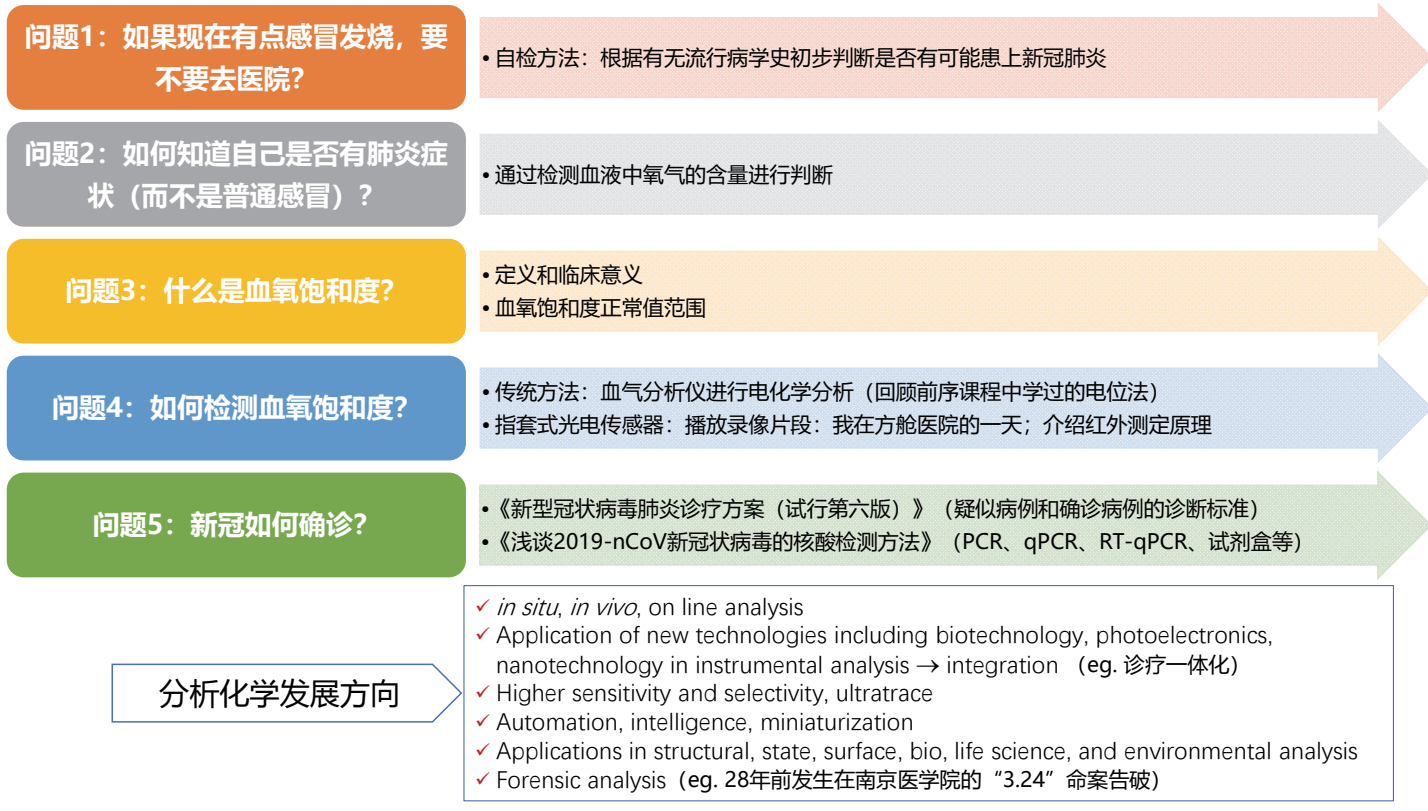

图 1 微课 “从新冠肺炎的爆发看分析化学的重要性” 教学设计

\section{3 以奎宁为出发点, 讲好化学 “抗疫” 故事}

进入 3 月, 随着疫情在全世界逐渐蔓延, 各种诊疗方案的讨论在网上持续发酵, 以氯喹为代表 的人工合成的系列抗疮药物, 先后被多国列入新冠肺炎的临床治疗方案中 ${ }^{[5]}$ (之后又有许多关于这些 药物副作用的报道); 同时, 随着课程的深入, 从 3 月下旬开始, 我们进入到 “分子发光光谱” 这一 章节的讲解。两者的交汇点是天然抗疮药物分子奎宁。由此, 在讲到奎宁的荧光性质时, 除了通常 
的苂光光谱分析中激发光谱与发射光谱之间的关系这部分内容外, 我们增加了 “奎宁系列药物发展 史” 的简单介绍。从印第安人用金鸡纳树的树皮或根皮者的水治疗㾏疾, 到奎宁的提取和合成、氯 喹等系列药物分子的合成和治疗机理的研究等; 自然也要提到抗氯喹恶性疮疾在东南亚的严重扩散, 1964 年, 越南政府向中国请求帮助, “523 任务” (防治㾏疾新药研发项目)的实施和后来的屠呦呦发 现抗疮药物青蒿素，以及这一过程中分离分析的重要性。

4 月 1 日, 恰逢分子发光光谱这一章内容讲完的那天晚上, CCTV4 “国家记忆” 栏目播出了 “战 疫” 纪录片《抗疮疾》。该片对这段历史进行了更全面的回顾和更权威的解读, 其中也提到了 “金鸡 纳霜” 奎宁。后来了解到从 3 月 30 日起, “国家记忆” 栏目连续播出了五集 “战疫” 纪录片, 讲 述了中国人民为人类对抗鼠疫、天花、疮疾、血吸虫病、脊髓灰质炎等恶性传染病所做出的突出贡 献。学生们从 2 月下旬开始, 虽然在家, 但也参与了 “抗疫”, 也有必要了解这些历史, 记住其中 包含屠呦呦在内的名字, 如 “鼠疫斗士伍连德” , 建国七十周年之际被授予 “人民科学家” 国家荣 誉称号的顾方舟。于是，我给学生布置了一个视频作业:

轻松学习时刻, 推荐大家观看 CCTV4 3 月 30 日至 4 月 2 日 “国家记忆” 栏目的“战疫”系列, 并完成以下课程小测:

1) 人类第一个全合成的复杂天然产物是什么?

2) 诺贝尔奖历史上有几次授予与疮疾相关的成果，请分别列出;

3) 人类历史上第一次依靠科学手段在人口密集的大城市成功控制传染病是哪一次?

4) 为什么在一定范围内燃放烟花爆竹相当于一次消毒消杀?

5) 结合所看的内容, 提一个与化学相关的问题。

一个荧光分子奎宁, 在以前的课上最多一、两张图片带过, 这次结合疫情的发展, 在课内、课 外拓展了许多内容。一方面使课程内容更加丰富多彩, 教学方式更加灵活多变, 增加学生的学习兴 趣; 同时也是为了培养学生对专业的热爱和认同感, 进一步认识到化学对于国家民生和人类健康的 重要作用; 更重要的是, 把课程内容和 “抗疫” 的现实相结合, 深入了解我们国家历次抗疫的过程 和科学家在其中的无私奉献, 学生应该更能理解国家和人民在这次抗击 “新冠肺炎” 中所做的巨大 牺牲和战胜疫情的来之不易, 更能分辨网络上的真伪信息; 有助于激发学生的民族自豪感, 和用自 己的专业知识投入到祖国建设中的热情。

\section{4 网络视频等多元素材的应用}

直播课一般要求提前几分钟开启直播模式, 等待学生们陆续进入直播课堂。利用这几分钟, 以 及课间, 可以给学生播放一些与教学内容相关的视频, 或者虚拟实验的展示。比如在红外光谱部分, 我们选取了 Berry Pseudorotation、ATR-FTIR、迈克尔逊干涉仪等网络上公开发布的视频; 分子发光 光谱这一章中, 向学生重点推荐 Fluorescence Animation、绿色荧光蛋白细胞定位等视频。视频的选 取原则一般是 5 分钟以内, 以英文视频为主，保证科学性的前提下，讲清楚一个问题。这些小视频， 可以在课前、课后播放，作为课程内容的衔接和过渡; 或者直接插入课程直播过程中，作为课程内 容的补充或知识点的强调。也可以向学生推荐一些学术大家的讲课视频, 比如华人诺贝尔奖获得者 钱永健先生亲自讲解的 “绿色荧光蛋白的发展史” [6], 时长 34 分钟。课后我们也会及时把视频链接 发在课程钉钉群上, 供有兴趣的学生自学。

除此而外, 在直播过程中会向学生解读最新文献, 以及一些相关的网络文章。比如最近的一次 是向学生介绍日本抗疫经验 ${ }^{[7]}$, 如轻巧便携的小型 ECMO 的研发、电梯等公共设施利用红外传感器 实现免触碰操作、从约 2000 种现有药物中用人工智能寻找新冠的临床治疗方法等。通过这些信息的 分享, 一方面希望能够提高学生查阅文献的能力; 另一方面希望学生能够及时了解最新的分析化学 特别是仪器方面的发展, 与导论课中的内容 “遥相呼应” ; 并强调开发具有自主知识产权的分析仪 器的重要性。 
在线下课程中, 视频等素材的灵活运用也非常重要, 可以在丰富课程内容的同时, 吸引学生的 兴趣。相比较而言, 线上直播课中, 教师和学生都直接面对电脑屏幕, 可以更加有效地观看视频、 捕捉其中的有效信息; 针对学生们远离校园、不能进实验室做实验的现实困难, 利用视频、动画、 虚拟实验, 能够使学生清楚直观地 “看” 到仪器的内部结构以及操作过程, 有利于学生更好地理解 方法原理和仪器结构; 在播放的同时, 教师加以适当的解释和点评, 及时提问启发学生思考, 则听 课效率可以进一步提高。总体而言，在线上学习阶段，有效地运用网络多元素材，更显重要。

\section{5 结语}

网络直播教学, 教师可以脱离传统备课的刻板, 利用丰富的多元化素材和师生互动的教学方式 配合知识点的讲解; 可以进一步提高学生的学习兴趣, 有利于学生理解原本枯燥的 “仪器、化学结 构和数据” , 帮助学生更好地理解分析化学的专业知识; 将疫情相关内容有机融入教学过程, 更是 分析化学课程的独特优势。

新冠肺炎的爆发, 给了高校和广大教师一个重新认识、建设线上课程的意义和难得的全面线上 教学的实践机会。在线技术让网课变得生动活泼, 但最基本的要求是教师教育理念的真正革新, 给 课程带来由内而外的教学突破。在传统课堂与在线课堂将长期共存并深度融合的状态下, 如何加快 在线教学由 “新鲜感” 向 “新常态” 的转变 ${ }^{[8]}$, 将线上教学的优势与线下教学有机结合, 让学生成 为真正的学习中心，调整学习策略和提升学习能力，是接下去高校陆续复课、恢复正常教学后我们 仍然需要思考和不断实践的问题。

\section{参 考 文 献}

[1] 仇子龙. “知已知彼：关于病毒, 这些秘密你必须知道”. (2020-02-07) [2020-04-30]. https://tech.sina.com.cn/scientist/2020-02-07/dociimxxste9467544.shtml.

[2] Moolnet. “浅谈 2019-nCoV 新冠状病毒的核酸检测方法”. (2020-02-10) [2020-04-30].

https://mp.weixin.qq.com/s?_biz=MzU5OTE2NDc0NA==\&mid=2247484623\&idx=1\&sn=89e83cfbd70145ed04a68f0debace22a\&chksm=feb85 6e8c9cfdffed8afe68500271 cb39918b2888bbd85bc4e02f1 f2eb1143ad5cb25ec6f435\&mpshare=1\&scene=1\&srcid=\&sharer_sharetime=158132702 0490\&sharer_shareid=009513b83c179d7512a37a6f6b66276c\&exportkey=AekwqCFRRG5b0080d672B38\%3D\&pass_ticket=W82BI\%2BxCUFrd uM7mO2qabYaZrxxaP1KsBhWkO0LN853g\%2F0\%2FEXfgowFadZaFI4R9s\#rd.

[3] 中国新闻社. “方舱医院的生活什么样？网友：太高能了！”(2020-02-19) [2020-04-30]. https://item.btime.com/f43jg9e1lv99lnpora2g1i2ek6n.

[4] 国家卫生健康委员会. 新型冠状病毒肺炎诊疗方案(试行第六版). 2020-02-19.

[5] 后山. “穷人的希望”一奎宁、氯喹、羟氯喹的前世今生. (2020-03-22) [2020-04-30].

https://mp.weixin.qq.com/s?_biz=MzI3MTIxNTgyMw==\&mid=2651589314\&idx=1\&sn=59f78974eb2a2bf63ca8a14d51c38b76\&chksm=f13d97 62c64a1e74d055b021e9flefcd 1b69b06057f9b99e8140a32d05be8ald41f151df442e\&mpshare=1\&scene=1\&srcid=\&sharer_sharetime=158486354 8017\&sharer_shareid=27ba3d01 f6b425baa532d6a0243dd3c7\&exportkey=AZYtwF9KvoMDLuAJIVI56TQ\%3D\&pass_ticket=pB1INmHYz0e53 PjLydZkRSUHtXOcRKIrrhgxXMoRlbIpP2\%2Fa6c5XllFRSpHUKtZM\#rd.

[6]【生物医学大讲堂】钱永健-绿色荧光蛋白的发展史. [2020-04-30]. https://v.qq.com/x/page/r0325gi98ob.html.

[7］博士学者圈. “凭什么日本至今也没挤爆医疗系统？因为我们只会补救，他们却在创造”. (2020-04-19) [2020-04-30]. https://mp.weixin.qq.com/s?_biz=MzI3OTQ3OTg1MQ==\&mid=2247492212\&idx=2\&sn=951906b75cd9e543bfb001e65dc04732\&chksm=eb45 b130dc323826162ad0665f01dbd1b0bb9fe400a7b91b5c8373ed01bc093a70d7cf4148e0\&mpshare=1\&scene=1\&srcid=0421L4SS6VtiXY5vgVNhS Cwk\&sharer_sharetime=1587806272159\&sharer_shareid=7f15a3aa76e224d4a269989fead27277\&exportkey=AQUFxFO5nVZ51MaJwLgknmk\% 3D\&pass_ticket=YImJvcSFbSLjFymY2A5zpyFDc2xSIGgIdiptuvaKBXltHUfoTJCjXDXWHHIDouTI\#rd.

[8] 吴岩. 以学习革命推动质量革命一一在高等学校在线教学国际平台上线仪式暨“爱课程”国际平台发布会上的讲话. (2020-04-28) [2020-0430]. http://my-h5news.app.xinhuanet.com/h5/article.html?articleId=c9ea2cd5e31627e6d5a3d5714f095ffa\&share_device_token=7DAD3364-D57447C6-A17D-1782DB6EE822\&share_time $=1588046906579 \&$ share_type $=1$. 